\section{Why is it Difficult to Simulate EBSD Patterns Accurately?}

\author{
Alwyn Eades and Andrew Deal \\ Lehigh University, Bethlehem, PA \\ jae5@Lehigh.EDU
}

Electron backscattering diffraction (EBSD) has become a technique of great importance in scanning electron microscopy. The diffraction patterns obtained by this method are of value in identifying phases and in determining the orientation of samples. The technique was initially developed about thirty years ago and has enjoyed widespread use in the last decade or so. It is therefore something of a surprise that there do not exist computer programs that simulate the patterns well. In fairness, we must make it clear that a number of programs exist and others are being implemented, which represent important steps towards the simulation of EBSD patterns. However, as we hope these notes will make clear, a satisfactory simulation will be very difficult and we are not aware of any existing or forthcoming program that does a job that can be guaranteed to give accurate values for the intensities in the experimental patterns. Programs to simulate patterns in transmission microscopy have been in general use for a very long time and are in close agreement with experiment. This note serves to explain why it is so much harder to simulate EBSD patterns and to offer a possible framework for implementation. We hope that someone will take up this challenge in the near future.

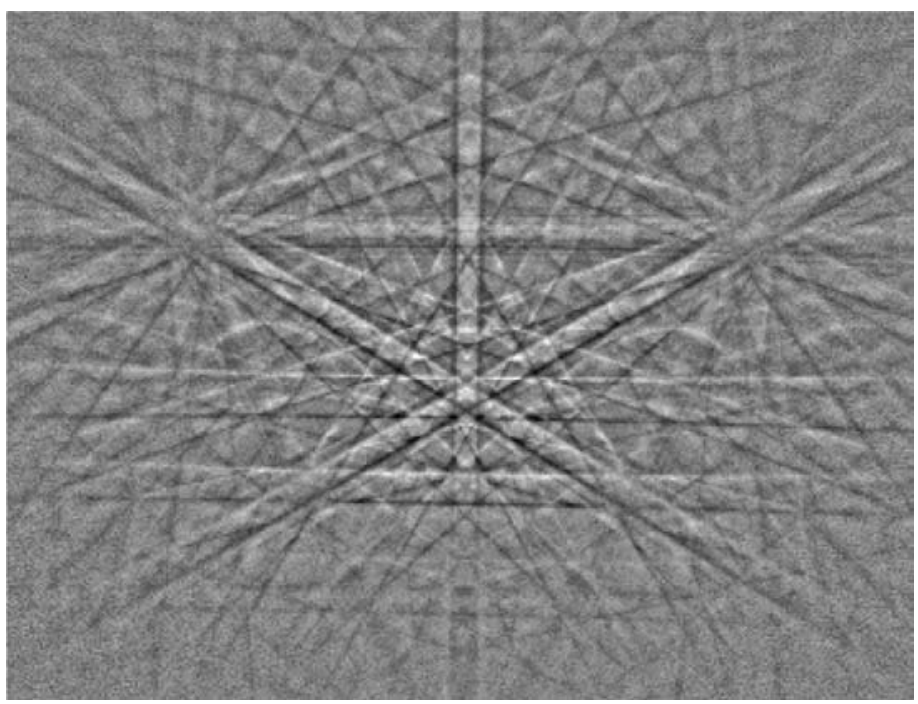

Figure 1 Characteristic appearance of an EBSD pattern, in this case from silicon. The bands crossing the pattern, called Kikuchi bands, are the result of the diffraction of the backscattered electrons as they leave the sample. They lie along the projections of the planes in the crystal.

There are a number of publications in which people have simulated electron channeling patterns. We have heard people say (wrongly, we believe) that EBSD patterns can be simulated in just the same way because there exists a reciprocity relation between the two techniques. We have spent some time thinking about how to simulate EBSD patterns and these notes are an informal expression of why we think the problem is hard and why the channeling simulations cannot be used.

In simulating channeling patterns, the electrons entering the sample are modeled - typically using a Bloch wave formalism, but a multislice method could be used-to give data on how the electrons channel as a function of the angle between the beam and the sample. This is translated to a mean depth of the first large-angle scattering

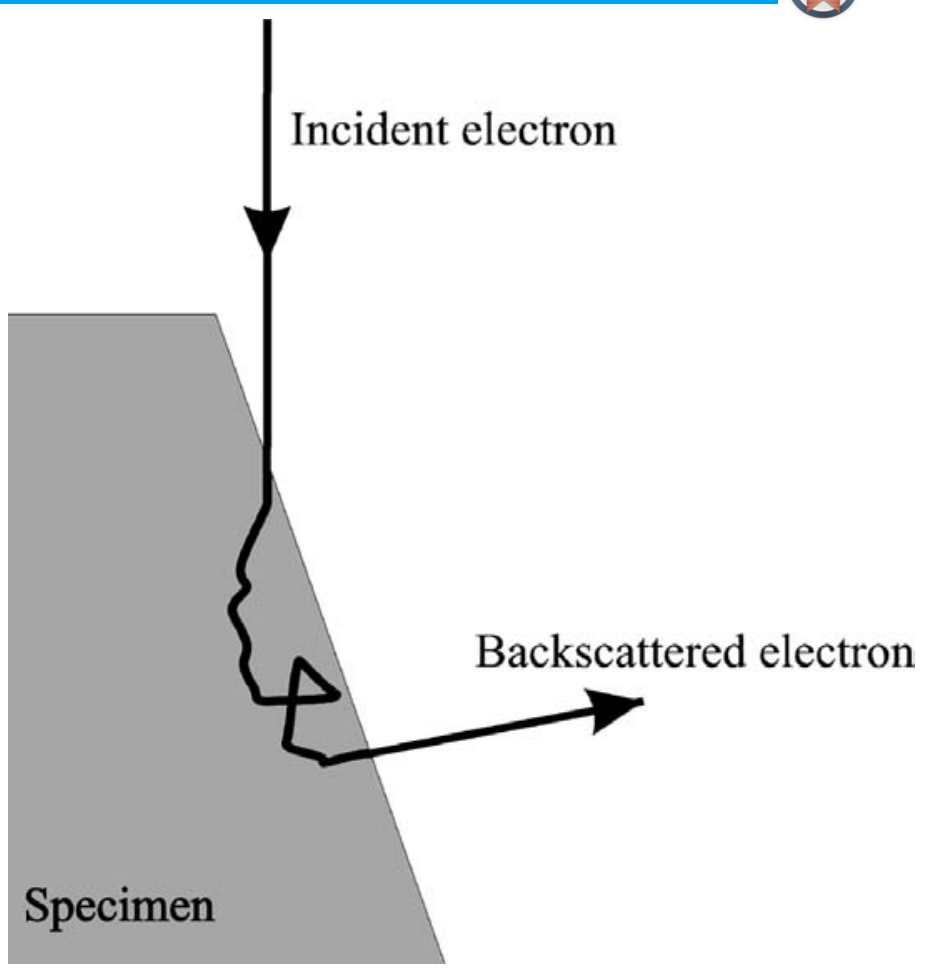

Figure 2 Schematic representation of the trajectory of a single backscattered electron. Each electron follows a different path because of the statistical nature of the scattering processes. The backscattered electron which contributes to the EBSD pattern has lost energy along the path through the specimen and each electron loses a different amount of energy. The dynamical diffraction process, which gives rise to the Kikuchi lines in the EBSD pattern, occurs as the electron leaves the sample. The energy of the diffracting electron is not the beam energy but the energy of the electron as it exits the specimen.

event (as a function of incident angle). A backscattered electron or secondary electron yield is then calculated using transport equations or Monte Carlo methods. No attempt is made to track how the crystal structure modifies the transport of the electrons beyond the first large-angle scattering event.

One could imagine that EBSD patterns could be simulated by doing the same thing in the reverse order. The incident electron beam would be simulated by Monte Carlo methods or by a transport equation approach, until the electrons are close to the exit surface. Once the electrons are close to exiting the sample, the diffraction effects would be simulated by Bloch or multislice methods as before. So why does reciprocity not apply? In the channeling case, the relevant diffraction effects all occur for electrons at the energy of the incident beam. There is no tracking of diffraction as the electrons lose energy. In the EBSD case, the electrons that exit the surface have a range of energies from the incident beam down to zero (or wherever one chooses to set the cut off), and all of these electrons are diffracted. To simulate the pattern it is necessary to perform the Bloch wave or multislice calculation for a range of energies from the incident beam energy down.

There is a second problem with the EBSD geometry. It relates to thermal diffuse scattering. In the channeling case it is usual to ignore thermal diffuse scattering except through its incorporation into the Debye-Waller factor. The fact that there may be thermal diffuse scattering along the initial part of the trajectory (modeled by Bloch methods) is assumed not to materially affect the depth of penetration to the first large angle scattering event and hence not to affect the probability of the electron being scattered out of the sample. 
By contrast, in EBSD, if there is thermal diffuse scattering of the electrons while they are in the channeling phase (just before exiting the sample), this will affect the diffraction pattern that is observed, by putting the electrons onto a different position on the EBSD screen. There is an analogous situation in TEM. A TEM diffraction pattern shows diffuse scatter in the space between the elastically diffracted beams. Kirkland, for example, has shown that this can be simulated by using a "frozen phonon" modification to multislice computations. There is a strong implication then that, to simulate EBSD patterns, the channeling at the exit surface needs to be simulated in the same way, which is with a dynamical diffraction calculation that keeps track of the direction of the electrons that are subject to thermal diffuse scattering. This could be done with a frozen phonon multislice calculation. Alternatively, it may be possible to incorporate thermal diffuse scattering into a Bloch wave calculation but we are not aware of it having been done.

Therefore, it would be necessary to do a dynamical calculation incorporating the effects of thermal diffuse scattering for each different energy and each different exit orientation of the electrons and in each case for a sample thickness big enough to cover the deepest depth from which electrons could exit the surface without further large-angle scattering. This would make the calculation horrendously large even by the standards of today.

There are some other technical problems that need to be addressed. They may be tricky in practice but should not be difficult in principle. One is that the multislice method as conventionally used for TEM calculations cannot handle large angles (neither large angles with respect to the surface, nor large angles with respect to the incident direction). Modifications would be needed to handle both kinds of large angles correctly. Some have already been proposed, but not applied to the problem at hand. There is also the matter of matching the output from the Monte Carlo calculation to the input to the dynamical diffraction calculation. The electron path from the last large-angle scattering event to the sample surface, as determined by the Monte Carlo calculation, could be used as the path for a single diffraction calculation. Statistics of the electron trajectories, energies, and diffraction path lengths could be generated by Monte Carlo and then used to determine the appropriate diffraction calculations to perform. These could be computed, statistically weighted, and collectively combined to simulate an EBSD pattern. Savings in computational time might be realized by carefully binning the energy, trajectory, and path length ranges.

In summary then, there are very good reasons why there is not yet an EBSD simulation program that can be considered satisfactory. To solve the problems outlined in this article, two approaches seem possible. First, one could write a program that does the complete calculation. It would be a huge computation and would take a very long time even for the computers of today. Alternatively, one could look for simplifications and approximations to make the computation tractable. However, it would be necessary to show that any such simplifications would not make significant changes to, for example, the profile of the Kikuchi bands (since the profile might be used to extract information on the lattice parameter). This is a real problem that has not yet been solved. Kinematic calculations do not accurately predict the high intensity Kikuchi bands seen in many engineering materials. This makes phase identification and automated mapping difficult for many new materials without manual scrutiny. We submit this note in the hope that someone will take up the challenge posed by these problems.

\section{The New Dual Standard.}

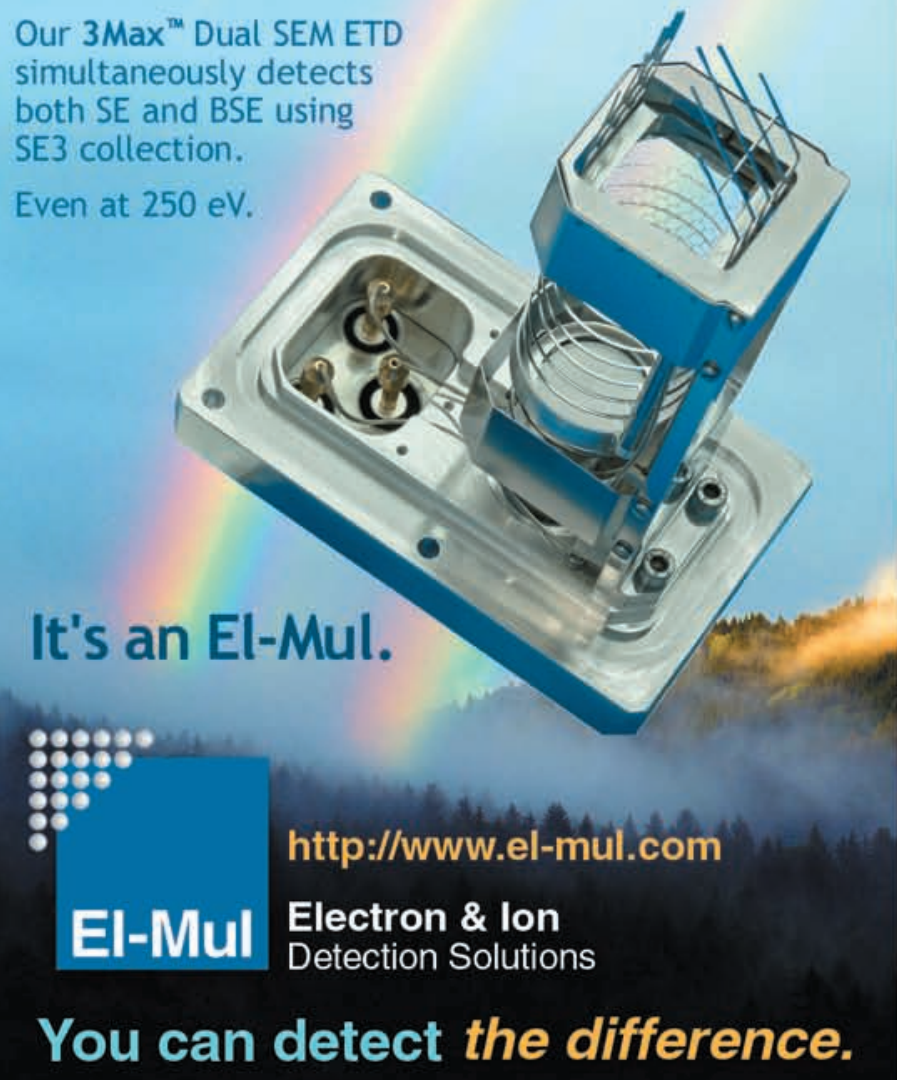

INDUSTRY RESEARCH MEDICINE

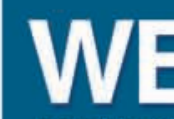

BREAKTHROUGH TECHNOLO

High Resolution Imaging

of Fully Hydrated Samples

Greatly Reduces Prep Time

Capsule Fits Standard SEMs

Safe and Simple to Use
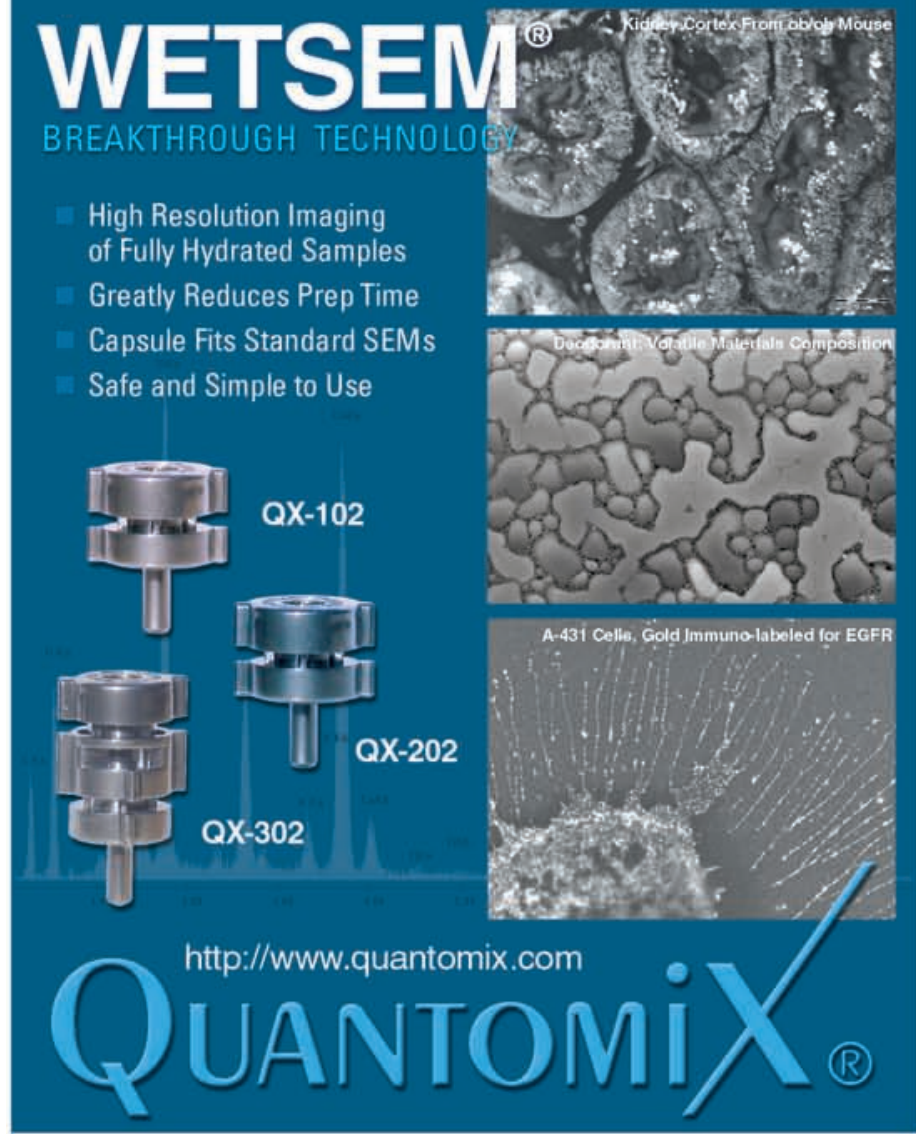

\section{QX-302}

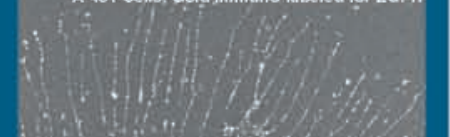

तो 702

http://www.quantomix.com
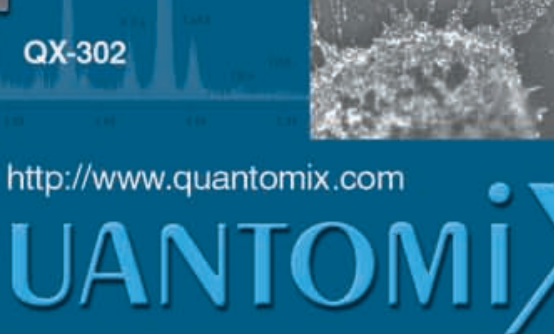